\title{
Designing Module E-Complaint System Based on Geotagging and Geofencing
}

\author{
https://doi.org/10.3991/ijim.v11i3.6557 \\ Puspa Miladin Nuraida Safitri A Basid \\ Universitas Brawijaya, Indonesia \\ Puspamiladin2808@gmail.com \\ Herman Tolle \\ Universitas Brawijaya, Indonesia \\ emang@ub.ac.id \\ Fatwa Ramdani \\ Universitas Brawijaya, Indonesia \\ fatwaramdani@ub.ac.id
}

\begin{abstract}
The government initiative in the implementation of smart city concept is an effort in the management of a city. In the governance of a town, public service is one of the most important aspects. Improving public services requires public role because the society is the direct users of public services. One role of the community in improving public service is to provide information or complaint to the government. However, the media where the public reports such information has yet been optimum. Therefore, the researcher assumed that the public needs certain media as well as some support system where they can make complaints related to public service. The support system is in the form of e-complaint system installed on smartphones equipped with geotagging techniques to determine exactly where the complaint is made (location) and geofencing method to determine which territory the complaint belongs to. In this study, the researcher explained the process of designing module for geotagging and geofencing-based e-complaint system. Several experiments were conducted to find out the accuracy of these techniques. With $94 \%$ accuracy, geofencing was a suitable technique to apply. In addition, geotagging techniques using EXIF of a digital image was considered the most suitable technique for the e-complaint system rather than other geotagging techniques.
\end{abstract}

Keywords-geotagging, geofencing.

\section{Introduction}

Recently, several cities in Indonesia has began to apply the concept of Smart City that refers to a concept to improve the quality of people's lives by making use of information and communication technologies in the management of a city [1]. One effort in the management of a city is improving public services. The 2009 Decree 
number 25 on Public Service states that one of the government's obligations is to serve all citizens and residents so that their basic need is met and their rights are fulfilled within the framework of public service which is mandated by the 1945 Constitution [2].

Improving public services requires active participation of the community since the public is the one using public services. In other words, public participation in improving public services is pivotal. One example of the public participation is public complaints. Public complaint is an indicator how much the public care about qualified public services. However, it is undeniable that the media where the public can make a complaint has yet been optimum. Very few people know how to make complaint related to public service properly. The government has launched website where the public can make complaint for example the website of East Java government (http://jatimprov.go.id). However, the researcher thought that the method has yet been effective for the public to deliver their complaints. In addition, the website has several limitations. Before filing their complaints, the public should write down their complaint manually. In conclusion, the recent public complaints management has yet been able to cater public need.

Based on the problems above, the researcher concluded that a facility or media as well as some support systems where public can make complaints more effectively is needed. The researcher is interested in conducting a study related to E-Complaint System based on Geotagging and Geofencing The purpose of the study is to design and develop more efficient media (smartphone-based application) where public makes complaint. The researcher decides to develop application for smartphone due to the number of smartphone users in Indonesia. According to eMarketer, by 2015, there were 55 million smartphone users in Indonesia [3]. The users can potentially contribute in developing the quality of public service.

One of the methods used in the study was GPS (Global Positioning System) sensor available in cellular phones. GPS determine one's position based on signals received from the satellite smartphone. In other words, the satellite transmits the signal identifier, position and timestamp of the device [4]. Using GPS, the public can provide information such as images and the coordinates where problems related to public service take place. Geographic information such as longitude, latitude and altitude can be added to the image [5]. The addition of location information in the image is called Geotagging [6]. Geotaging can be done by adding information via Exchangeable Image File Format (EXIF) of an image. The information is stored in the EXIF header [7]. As an addition, adding geographical information can also be done separately via webservice.

Basically, these sensors are widely used by location-based service providers for searching for a location to navigation system. However, they are not only limited determine location in realtime. GPS also has other feature namely Geofencing. The feature is a type of technology for monitoring mobile devices in which the coordinates of the location are automatically sent to the control center. Monitoring position on this feature uses a set of geographical coordinates that will be used as virtual boundary (geofence) [8]. The shape of the virtual boundary is constructed in the form of a polygon that resembles the outer side of a region. Then Raycasting method is used to 
determine the existence of the coordinates. It will detect these coordinates by establishing an imaginary horizontal line to determine the lines that intersect with the polygon.

Before the application is launched, some tests are conducted. They aim at finding out whether the method is applicable in the e-complaint system. In the early stages of geofencing method, the researcher describes the implementation of the method starting from how to build a polygon to how to determine the existence of a point on the polygon. Then the researcher also describes the accuracy of the method using Raycasting. In terms of geotagging technique, the researcher explains how to apply the more efficient technique geotagging for the e-complaint system. So, both of these methods can be applied eventually. The purpose of the study is to determine the design of the module for E-Complaint system with geotagging and geofencing approaches.

\section{$2 \quad$ Literature Review}

Some studies related to these topics have been conducted previously. Bareth, Kupper, and Ruppel conducted a study in 2010 that proposed a new concept to distribute applications with geofence services [9]. It created a marketplace to acquire or subscribe applications. In general, keyword was needed to find certain application; however, application can also be found using geofence services. Their study was a prototype where the accuracy and use of the application has yet been tested thoroughly. Another study was conducted by Suyama and Inoue in 2016. Their study proposed a concept to provide information in the form of a notification when the users entered a geofence area [10]. The purpose of the notification was to inform the users who entered a disaster-prone area. As the result, it would minimize number of victims. The application covered the areas prone to disasters at that time. However, the accuracy of the geofence detection was considered quite low. The low accuracy happened because the application relied heavily on the internet. When there was not any internet in certain area, the application could not be used effectively and as the consequence influenced how accurate the information the application gave.

Sen conducted another research in 2014 [11]. The study used crowdsource as the primary method of traffic management application. The application called RasteyRishtey collected and shared GPS traces from the public about traffic condition. GPS traces left by the users were analyzed and turned into information about traffic conditions. The study only utilized geotagging technique to share the trail of coordinates.

Indonesia basically has had e-complaint system. Jakarta has application called Qlue for handling complaints from the community. The public can make complaint from their smartphones. They can also provide information such as images and text descriptions related to the complaint they make. In addition, geotagging features have also been incorporated in Qlue. The society can just download the application from app stores. However, anyone, including those does not live in Jakarta, can download the application. As the effect, the issues related to public service may happen in the areas other than Jakarta. Thus, a feature that can determine the coordinates of com- 
plaints and detect whether the location of the complaint is within or outside particular area of authorization is needed.

In addition, Qlue can only be accessed by users registered in the system. Unregistered users cannot find out about complaints the registered users make. The researcher considers limited access to visualization as a problem more particularly for regions with low response to public complaints. Thus, visualization that can be accessed by both e-complaint system users and non-users is of necessity. In the study, the researcher took advantage of GPS sensor for geotagging and geofencing techniques in e-complaint system and crowdsource as the method to collect data (public complaints). The system uploads the complaint and displays it in web visualization that that can be accessed by both users and non-users of the system. The researcher will also determine the accuracy of geotagging and geofencing techniques before being applied in this system.

\section{$2.1 \quad$ E-Complaint}

Complaint has different meanings in each field. According to the Business Dictionary [12], complaint is a statement filed by the complainant to another party as respondent. The statement was based on the recovery of assets such as money or property. When viewed from the standpoint of communication, the complaint is an active communication that could lead into an "interaction." The core of any complaint is to improve the user dissatisfaction into satisfaction. In addition, the government or any system needs feedback from the community to find out the shortcoming of a system [13]. Feedback from the public is regarded as complaints and suggestions, which can be considered to improve services. Then, it becomes an obligation to manage the complaint as appropriate. Currently the system to manage complaint from the public is still considered as conventional one. The conventional complaint process or one currently works recently is [13]:

1. Submit complaint directly to the complaints department.

2. Submit complaint to the specific organization in a government agency which is usually called Complain Handling Department.

The researcher considered the process ineffective. In addition, these activities require too much time. A complaint handling system with the integration of technology is relevance because it works more effectively and saves time.

\subsection{Geotagging}

Geotagging refers to process of adding information such as geospatial data in the metadata of a media be it a photograph or video. The geospatial information added is in the form of longitude and latitude [14]. The purpose of the process is so that the object (media) can be positioned exactly to the coordinates on a map [15]. There are several methods to add geospatial information, namely: 
- Insertion of information via EXIF Exchangeable Image File Format (EXIF) is a specification for images with image formats such as JPEG and TIFF [7]. EXIF contains some information such as camera settings, the environment where a picture is taken, the picture itself as well as copyright information and geographical information [6]. Other geographical information such as longitude, latitude and altitude can also be added to the image file [5]. The information is stored in the EXIF header [7].

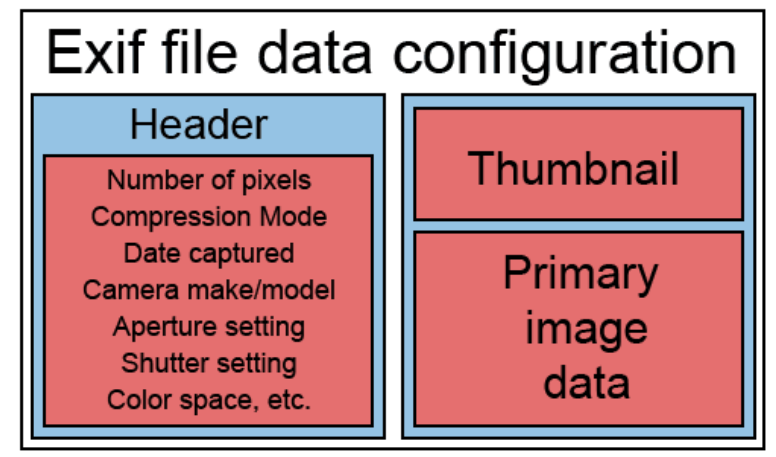

Fig. 1. Structure of EXIF (adapted on [7])

- Insertion of information via webservice. At this stage, information/ data such as longitude and latitude is not inserted in the EXIF format. Instead, information about location information is requested by posting the information on the webservice. Then, the pictures and information are uploaded separately into image available in the server.

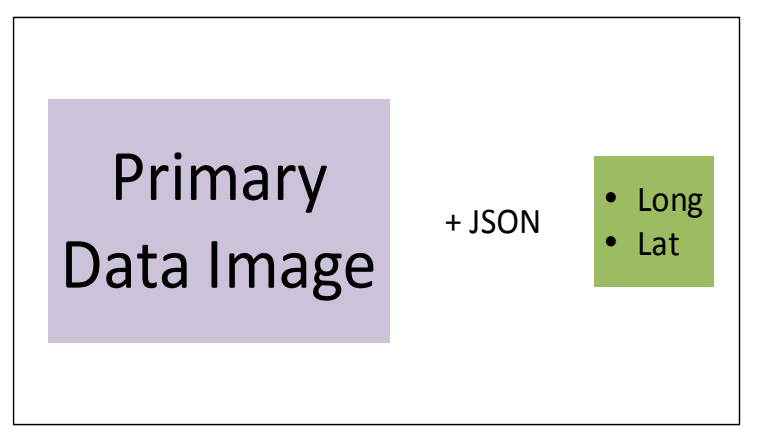

Fig. 2. Adding the information using Web service

\subsection{Geofencing}

Geofencing is a technique that is frequently used for location-based systems recently. In certain problems, Geofencing technology is used for monitoring moving objects by using the GPS sensor. In monitoring an object, geofencing works by build- 
ing a geographic area that is surrounded by a virtual fence (geofence). A set of geographic coordinates are used to form a virtual fence (geofence) about the geographical area [8]. Various techniques of Geofencing have been developed to meet different needs, such as Geofence area [8]. The shape of Geofenced area can be a simple as a geometric square or more complex shapes such as polygons.

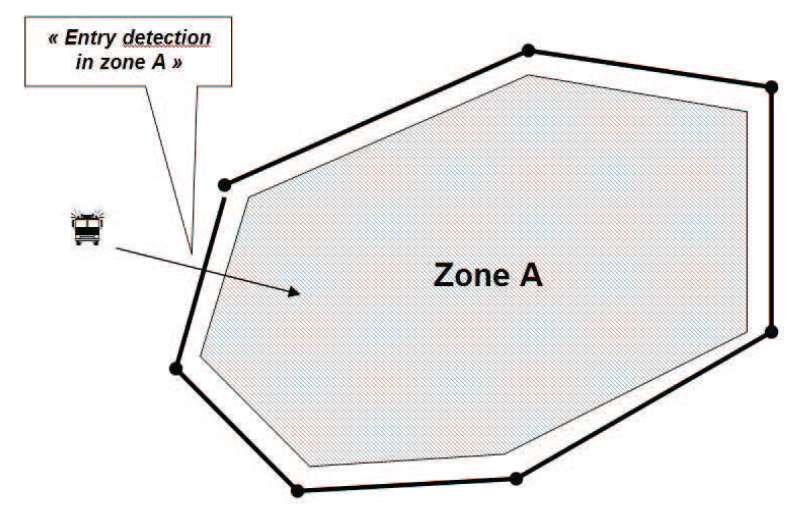

Fig. 3. Geofenced area (adapted on [8])

Polygon can represent one into a two-dimension form. It is used to represent buildings, farms, and local district administration [16]. Using polygon, one can get information about the whole shape of an area.

- Creating a polygon on a digital map: Making the polygon as a virtual fence on a digital map can be done by collecting the coordinates as a point. When a set of point coordinates has been collected and arranged in a certain order, it will create polygon feature. In creating polygon area, the first and the last coordinates should be the same. The remaining coordinates should be unique or different from one another [16].

\section{Vector area}

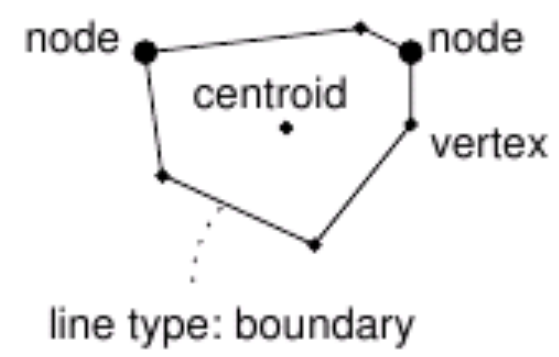

Fig. 4. Polygon Area (adapted on [12]) 
- Determining the position of the point using Raycasting:

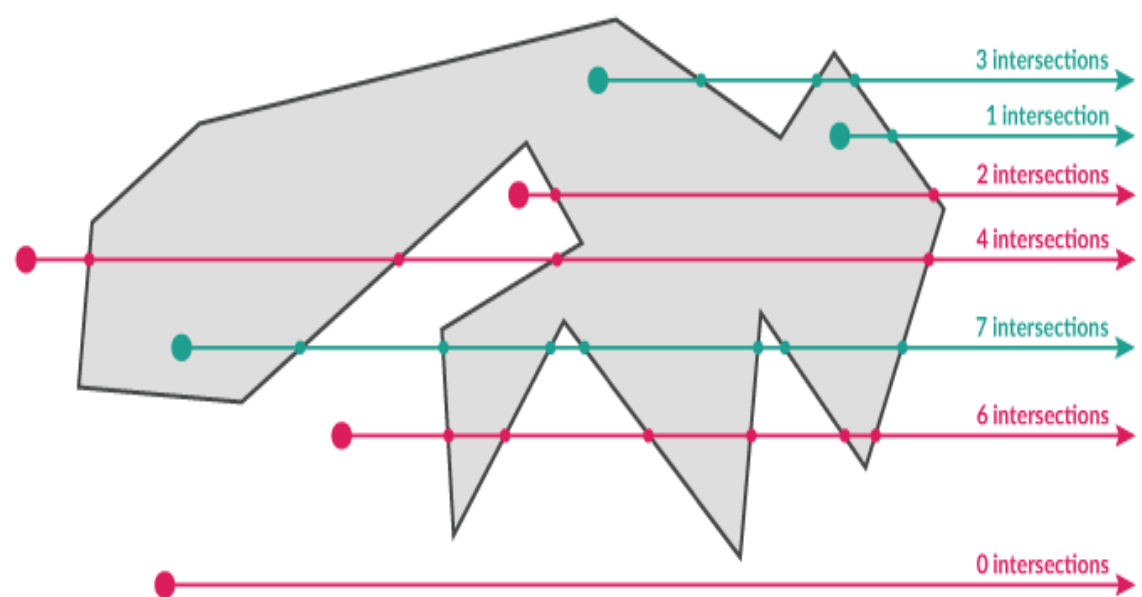

Fig. 5. Raycasting method (adapted on [17])

Raycasting method is a method for determining whether user is inside or outside polygon area. Before determining the user's position, the user inputs some data namely coordinates detected in geotagging process. Result from the previous geotagging process is then detected to determine whether the user is inside or outside the polygon area. The coordinates are detected by drawing imaginary horizontal line to determine the lines that intersect with the polygon. The purpose of the establishment of the imaginary lines is to find out how many sides of a polygon parallel to testing coordinates. For the coordinates inside the polygon area, the number of lines that intersect with the imaginary lines forms odd number. At the opposite, when the coordinates are located outside the polygon area, the number of lines that intersect with the imaginary lines forms even number [17].

\section{Proposed Model}

Figure 6 describes the architecture of the system in general. In the early stages, users took pictures and added geographical information about the pictures using geotagging method. In the geotagging technique, the researchers would select the most suitable social crowdsource-based e-complaint system for mobile devices. The geotagging technique used was geotagging through EXIF of an image and geotagging via webservice. In the result of the experiment section, the researcher discussed which method used in this system extensively. After selecting the most suitable geotagging technique, the next step was to analyze where coordinates are located. Geofencing method described previously is used in the process. The method used in this stage was Raycasting. The system was able to determine whether the coordinates were inside or outside geofence areas. When the coordinates were outside geofence area, the images the users uploaded could not be displayed on the digital map of the entire upload. 


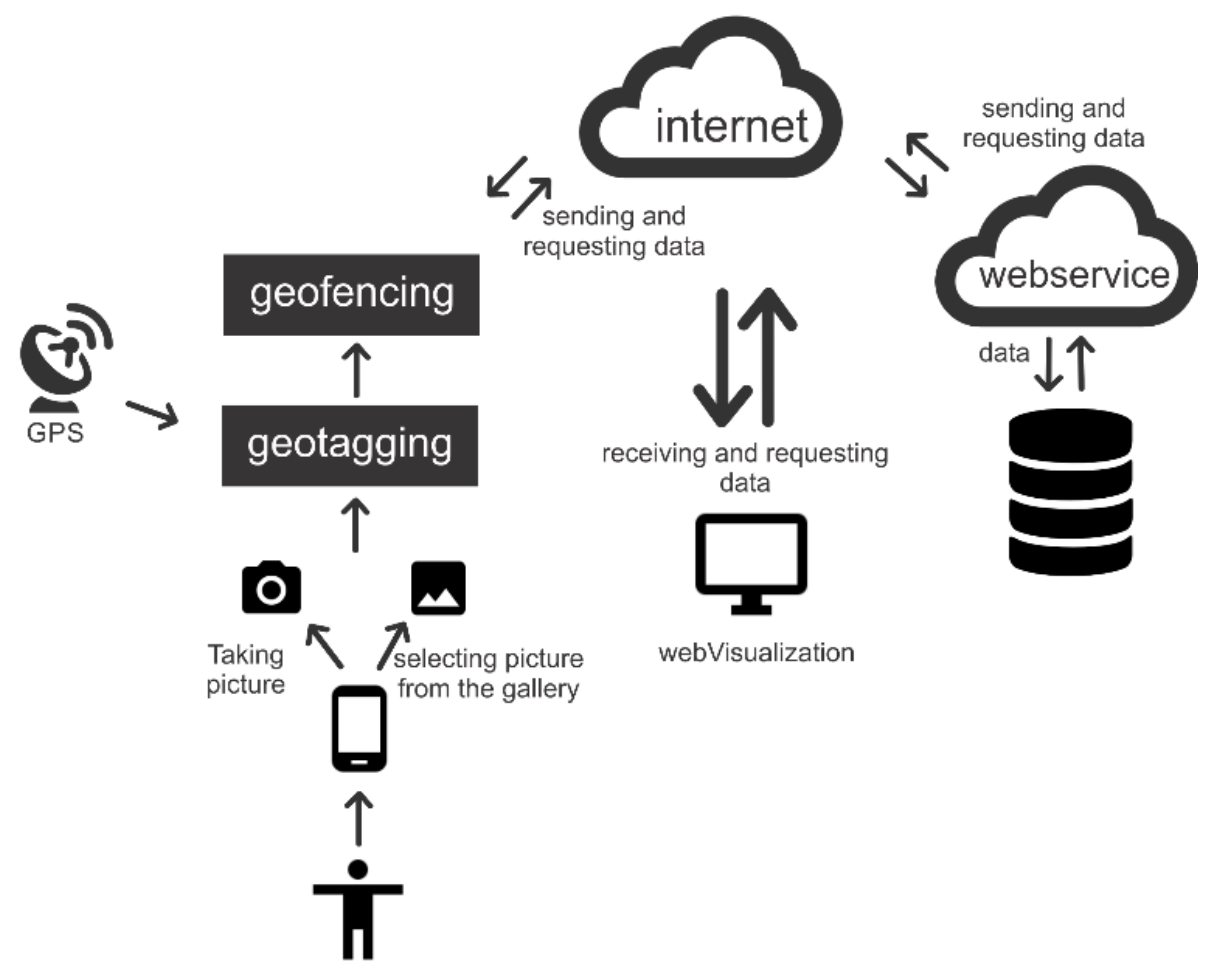

Fig. 6. Architecture of the System

Once the previous stage had been completed, the following step as the major focus of our future research is develop e-complaint system. Besides that, the system use crowdsource technique to collect complaints from the society. Then upload the complaint information into the server. When the complaint had been stored into the server, the information would be posted on the users' social media. The users would share the content on their social media. The purpose of content sharing/ complaint sharing was other social media users who were not the members of the e-complaint system may be aware of the complaint. It was expected that the content/ complaint would be viral on social media once it was uploaded. In addition, the benefit of sharing content via social media was faster response from the government. In addition to sharing content on social media, all complaint the users would be displayed on a digital map accessible for anyone. The image related to the complaint would be positioned according to the coordinates on the digital map. It was expected that the government would find the specific location where the complaint was made. 


\section{$4 \quad$ Implementation}

\subsection{Creating Polygons}

Several steps should be conducted to apply geofencing method. The first stage was to create virtual boundary as the barriers of an area. There are several types of virtual boundary. The shape of the virtual boundary relied upon which technique used. Some geofencing techniques such as proximity to a point of interest, route adherence, and route and schedule adherence requires a circle as the shape of the virtual boundary [8]. On the other hand, the technique of Geofenced requires polygon as the shape.

Figure 7 described the process of making a polygon on the system. The first stage was to determine the coordinates to form a polygon. These coordinates are determined from the outer limits of a region. In the system, the boundaries of a city were used as the coordinates to form the polygon as geofence area. The coordinates were stored in an ArrayList (point).

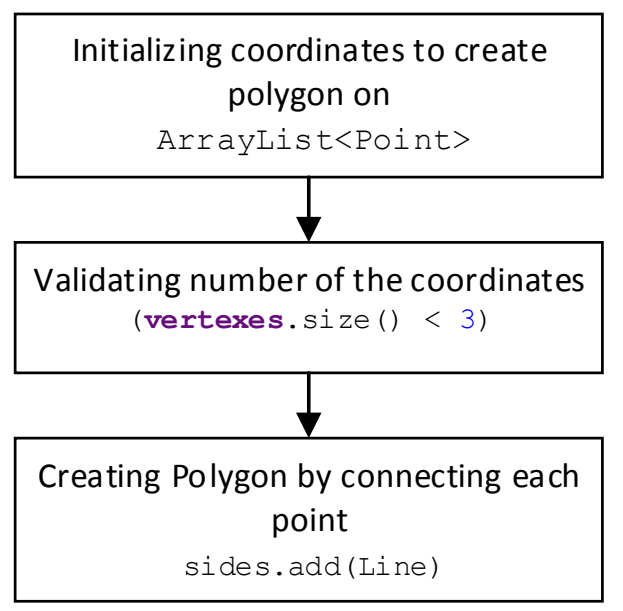

Fig. 7. Creating Polygon

The following stage was validating the number of coordinates stored in the ArrayList. When the total number of the coordinates was than 3 , one could not form a polygon. However, when the total number of the coordinates was more than 3 , one could proceed to the following stages. The third stage was to connect each of the coordinates based on the index in the Arraylist. By connecting each of the coordinates, the polygon as virtual boundary of certain geofence area was formed.

\subsection{Determining Pointer Position using Raycasting Method}

After successfully building a virtual boundary in the form of a polygon as the geofence area, the following stage was to determine the position of a point. It decided whether the point was located inside or outside the geofence area that had been estab- 
lished earlier. In order to do that, Raycasting method was used. In general, the method drew a line to determine the position of the point against the virtual boundary line. The process was described in Figure 8.

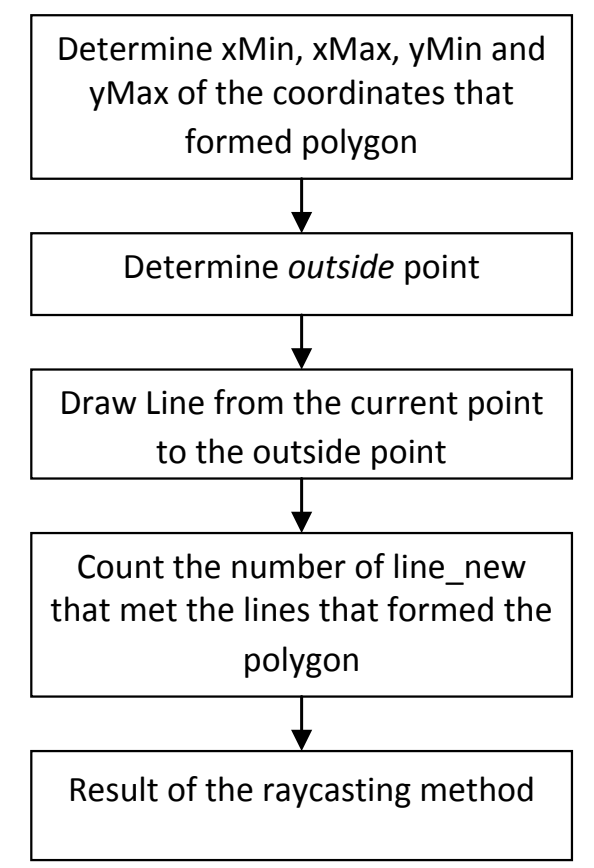

Fig. 8. Raycasting method

The first stage to determine the location of the point was to determine $\mathrm{xmin}$, $\mathrm{xmax}$, ymin, and ymax of the coordinates that formed the polygon. The xmin, xmax, ymin, and ymax were used to determine outside point. The outside this point is a coordinates that later would be used to draw line from current point to the outside point. As what had been explained previously, Raycasting drew line from a point to later find out whether the line met the virtual boundary line of the polygon.

Having determined the outside point, the next stage is to make a straight line from the current point towards the outside point. The line was a reference to show the virtual boundary of the geofence area. Once the line had been created, the system would check whether the line met each of the lines that formed a polygon. Then, the number of intersected lines was counted. When it was an odd number, then the point could be detected in the geofence area. However, when the result was even number, the point was located outside the geofence area.

\subsection{Geotagging method}

The geotagging method analyzed in the study was one using EXIF and webservice. Both of them were analyzed to find out which was the suitable one for the system. 
- Addition using EXIF. Figure 9 described how the method worked. It started by reading the EXIF of an image using available function/ feature. After getting latitude and longitude of the EXIF images, the final stage was to add them to the database.

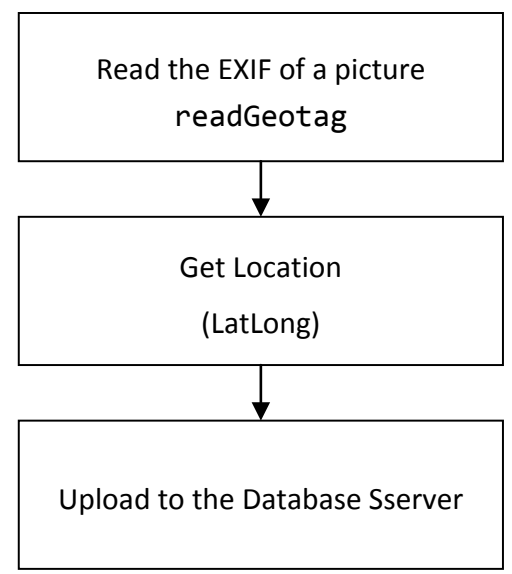

Fig. 9. Geotagging by EXIF

- Addition using webservice. To add information using webservice there were some additional process to do. The previous method of adding information was conducted only by reading the EXIF file to find out the coordinates; webservice was different from EXIF.

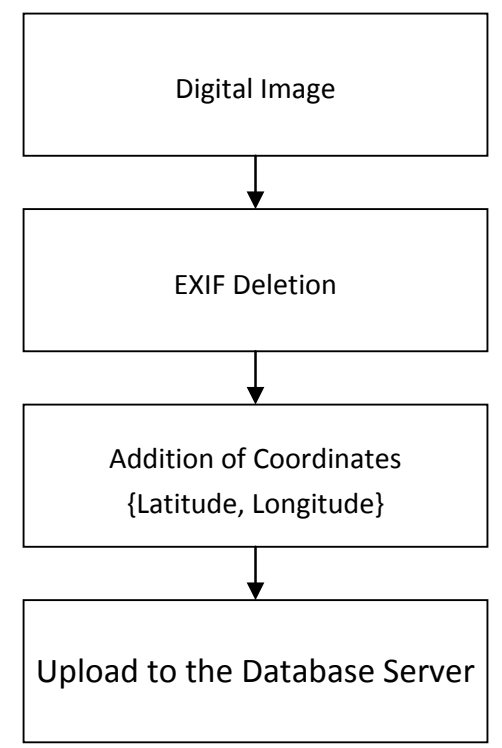

Fig. 10.Geotagging by webservice 
To add information about the coordinates of a location, the system should delete the EXIF file of a picture. It happened because each picture taken using digital camera had its own EXIF file. One should make another effort to get rid of the EXIF file of the pictures. Figure 10 described the entire process of adding information using webservice. The reasons why deleting EXIF file was necessary were described in the following section.

\section{$5 \quad$ Experiment Result}

\subsection{Raycasting Method Accuracy for Geofencing}

The testing scenario was accuracy testing. It was conducted to describe the accuracy of Raycasting method. The testing was conducted to 50 random points. Raycasting was then used to find out where the points were. Raycasting had $94 \%$ of accuracy. Raycasting could not detect where a point was when it was located on the border of a polygon area. In addition, another factor that influenced the accuracy of Raycasting method was how detail it was in describing points that created a virtual boundary. During the testing, Raycasting considered some coordinates located inside geofence area as areas outside the geofence. These were not the shortcoming of Raycasting but they would affect how accurate the system was. Table 1 showed 10 samples used in the testing. Out of 50 testing points, there were 3 times when Raycasting gave inaccurate result.

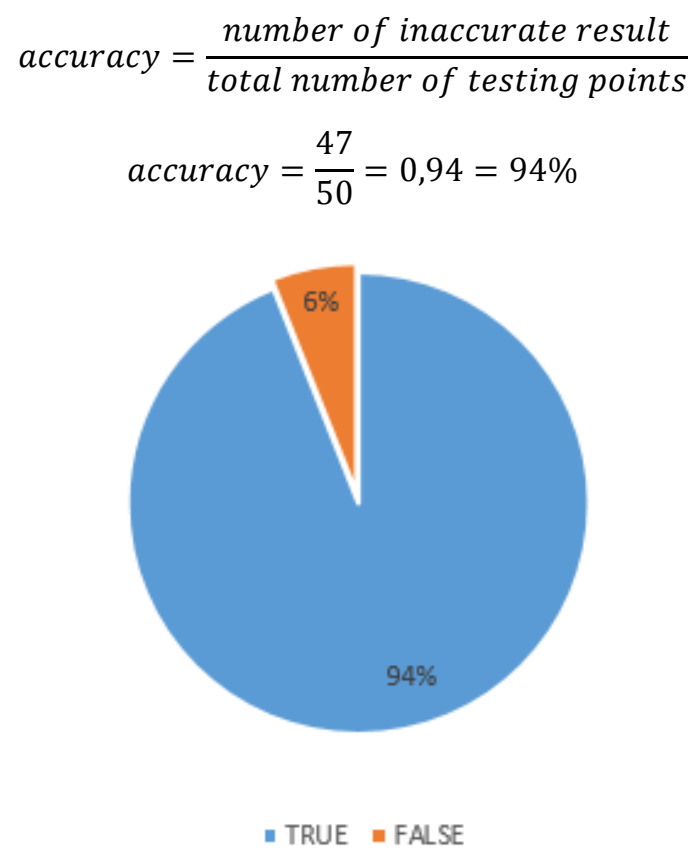

Fig. 11.Percentage of raycasting test 


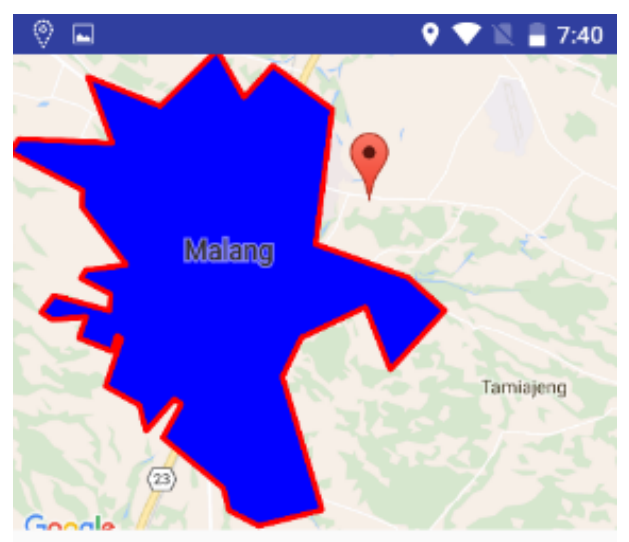

My Location :

Update lat/long :

$-7.9530886 / 112.6730206$

Status Anda :

Anda berada di luar Malang

SHOW LOCATION

\section{$\triangleleft$}

\section{O}

$\square$

Fig. 12.Raycasting Test

Generally, Raycasting was the suitable method to use in geofencing application on mobile devices. It was accurate, easy to use and delivered result in short time.

Table 1. Sample of Accuracy Testing

\begin{tabular}{|c|l|l|l|l|}
\hline \multirow{2}{*}{ No } & \multicolumn{2}{|c|}{ Coordinate } & \multirow{2}{*}{ T/F } & \multirow{2}{*}{ Status } \\
\cline { 2 - 3 } & \multicolumn{1}{|c|}{ Lat } & \multicolumn{1}{|c|}{ Long } & & \\
\hline 1 & -7.9682155891 & 112.6547470316 & True & inside the geofence area \\
\hline 2 & -7.9520383258 & 112.6608725646 & True & outside the geofence area \\
\hline 3 & -7.9528754855 & 112.6133652403 & True & inside the geofence area \\
\hline 4 & -7.9247377478 & 112.6341755642 & True & inside the geofence area \\
\hline 5 & -7.9127845618 & 112.6514345034 & True & outside the geofence area \\
\hline 6 & -7.9091243169 & 112.6777801662 & True & outside the geofence area \\
\hline 7 & -7.9466162091 & 112.6445928588 & True & inside the geofence area \\
\hline 8 & -7.9709287 & 112.6027745 & False & outside the geofence area \\
\hline 9 & -7.9137452793 & 112.6247901096 & False & inside the geofence area \\
\hline 10 & -7.9116591279 & 112.6280714571 & False & outside the geofence area \\
\hline
\end{tabular}




\subsection{Determine the effectiveness of Geotagging Method}

The second scenario was to find out how effective the geotagging techniques were. There were two techniques of adding information namely EXIF and web service. The researcher would compare processing time and size of data once additional information (longitude and latitude) had been added to certain pictures using the two techniques in order to select which one is the most appropriate technique.

During the test, the pictures were taken using Redmi 2 Prime cellular phone with 8 MPs camera. Geotagging was used to describe the effectiveness of each of the two methods. The results were described in Table 2.

Based on table 2, in terms of the size of data, webservice produced image with smaller size of data. In order to add EXIF information to an image, one should have $12.8 \mathrm{~KB}$ of data. On the other hand, webservice only required 40 bytes to add information about location.

Table 2. Sample of Geotagging test

\begin{tabular}{|r|l|r|r|r|}
\hline \multirow{2}{*}{ No } & \multirow{2}{*}{ Image } & \multicolumn{2}{|c|}{ With EXIF } & \multirow{2}{*}{ Without EXIF } \\
\cline { 2 - 4 } & & Original Size & \multicolumn{1}{|c|}{ EXIF size } & \\
\hline 1 & Test_1_Low.jpg & 553716 byte & 11718 byte & 541998 byte \\
\hline 2 & Test_1_Standar.jpg & 912228 byte & 12344 byte & 899884 byte \\
\hline 3 & Test_1_High.jpg & 1876310 byte & 11361 byte & 1864949 byte \\
\hline 4 & Test_2_Low.jpg & 1442369 byte & 21099 byte & 1421270 byte \\
\hline 5 & Test_2_Standar.jpg & 2551069 byte & 23453 byte & 2527616 byte \\
\hline 6 & Test_2_High.jpg & 4717692 byte & 22442 byte & 4695250 byte \\
\hline 7 & Test_3_Low.jpg & 563649 byte & 8886 byte & 554763 byte \\
\hline 8 & Test_3_Standar.jpg & 1078441 byte & 8693 byte & 1069748 byte \\
\hline 9 & Test_3_High.jpg & 2356320 byte & 8795 byte & 2347525 byte \\
\hline 10 & Test_4_Low.jpg & 644862 byte & 13715 byte & 631147 byte \\
\hline 11 & Test_4_Standar.jpg & 1065610 byte & 13193 byte & 1052417 byte \\
\hline 12 & Test_4_High.jpg & 2553873 byte & 13170 byte & 2540703 byte \\
\hline 13 & Test_5_Low.jpg & 606324 byte & 5744 byte & 600580 byte \\
\hline 14 & Test_5_Standar.jpg & 1498214 byte & 9471 byte & 1488743 byte \\
\hline 15 & Test_5_High.jpg & 2731486 byte & 7993 byte & 2723493 byte \\
\hline Rata-Rata & & 12805.13 byte & \\
\hline
\end{tabular}

However, another problem appeared when one wanted to build an application on a mobile device or smartphone. Digital camera embedded on the smartphone would automatically add information about camera settings and location/ coordinates to the EXIF of each image. When the researcher insisted on using webservice for geotagging, new problems would appear. In order to use webservice for geotagging on mobile devices, the system would require two additional processes. The first process was to remove the EXIF file from the picture and the second was taking existing coordinates, add them to an image and upload it to the server. 
The two additional steps above required more time. All mobile device users demand good responsiveness. Webservice for geotagging would take more time and therefore, it would not be able to meet the user's demand. As the result, EXIF was considered the more suitable method for smartphone-based applications, even though in terms of data size, webservice for geotagging was more effective than EXIF.

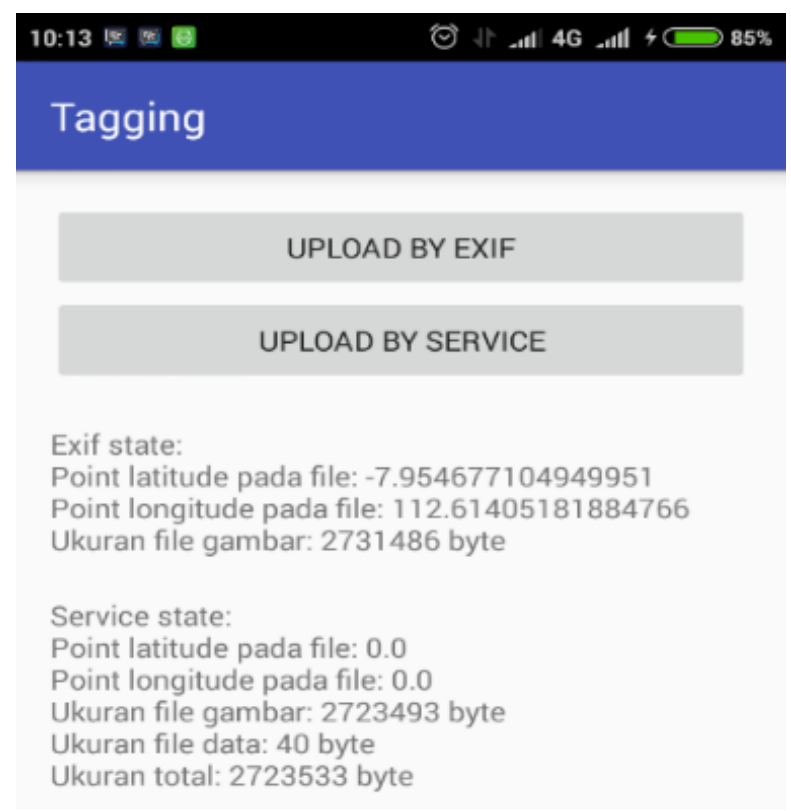

Fig. 13.Geotagging Test

\section{Conclusion}

Based on the tests, it can be concluded that Raycasting is an appropriate geofencing method for crowdsource-based e-complaint system. The accuracy rate was $94 \%$ based on the test to 50 random points. Raycasting has pretty short processing time that generates better responsiveness. As an addition, it is pretty accurate and therefore, suitable for the system.

The second phase is to test which method applicable for geotagging, the EXIF of an image or webservice. The finding is that webservice requires smaller amount of data. EXIF requires $13 \mathrm{~Kb}$ to store information about location and camera settings while webservice only requires 40 bytes to store information about location/ coordinates.

Even though the difference is significant, it is difficult to apply webservice for geotagging. Using webservice for geotagging will take longer processing time because one should delete the EXIF information attached to the images and add the coordinates. In conclusion, EXIF is considered the more suitable method for geotagging of the e-complaint system. 


\section{$7 \quad$ References}

[1] Nurrachman, K. I., "ITB \& Telkomsel Kerjasama Siapkan Smartcity" 2016. [Online]. Available: http://techno.okezone.com/read/2016/02/14/207/1311644/itb-telkomsel-kerjasa ma-siapkan-smartcity. [Accessed: 01-Jan-2016].

[2] Undang-Undang Republik Indonesia Nomor 25 tahun 2009 tentang Pelayanan Publik. [Online]. Available: http://www.setneg.go.id/index.php?option=com perundangan \&id=2274\&task=detail\&catid=1\&Itemid=42\&tahun=2009 [Accessed: 21 Juli 2016]

[3] EMarketer, "Asia-Pacific Boasts More Than 1 Billion Smartphone." [Online]. Available: https://www.emarketer.com/Article/Asia-Pacific-Boasts-More-Than-1-BillionSmartphone-Users/1012984. [Accessed: 22-Oct-2016].

[4] Schmidt, A. and Dannert., "Positioning Technologies and Mechanisms for mobile Devices,” Semin. Master Modul. SNET2 (TU-Berlin, ed.), 2010.

[5] Kakar, P and N. Sudha, "Authenticating image metadata elements using geolocation information and sun direction estimation," Proc. - IEEE Int. Conf. Multimed. Expo, pp. 236$241,2012$.

[6] Wu, M. Y., C. C. Hsu, and J. H. Lee, "A Geotagging scheme using image steganography and GPS information authentication," IIH-MSP 2009 - 2009 5th Int. Conf. Intell. Inf. Hiding Multimed. Signal Process., pp. 1245-1248, 2009. https://doi.org/10.1109/iihmsp.2009.301

[7] Japan Electronic Industry Development Association (JEIDA), "Digital Still Camera Image File Format Standard (EXIF) Version 2.1," June, vol. 12. p. 172, 1998.

[8] Reclus, F. and K. Drouard., "Geofencing for fleet \& freight management," 2009 9th Int. Conf. Intell. Transp. Syst. Telecommun. ITST 2009, pp. 353-356, 2009.

[9] Bareth, U., A. Kupper, and P. Ruppel., "geoXmart - A marketplace for geofence-based mobile services," Proc. - Int. Comput. Softw. Appl. Conf., pp. 101-106, 2010. https://doi.org/10.1109/compsac.2010.16

[10] Suyama, A. and U. Inoue, "Using Geofencing for a Disaster Information System," IEEE Comput. Soc. Int. Assoc. Comput. Inf. Sci., 2016.

[11] Sen, R., "RasteyRishtey: A social incentive system to crowdsource road traffic information in developing regions,” 2014 Seventh Int. Conf. Mob. Comput. Ubiquitous Netw., pp. 171-176, 2014. https://doi.org/10.1109/icmu.2014.6799090

[12] Vector Data, 2012. [Online]. Available: https://grasswiki.osgeo.org/wiki/Vector Data. [Accessed: 26-Sep-2016].

[13] B. Dictionary, “Complaint." [Online]. Available: http://www.businessdictionary.com/ definition/complaint.html. [Accessed: 27-Nov-2016].

[14] HosseiniM, K. Phalp, J. Taylor, and R. Ali, "The Four Pillars of Crowdsourcing: a Reference Model,” IEEE Eighth Int. Conf. Res. Challenges Inf. Sci., pp. 1-12, 2014.

[15] Miller, F. P., A. F. Vandome, and J. McBrewster, Geotagging. VDM Publishing House Ltd, 2009.

[16] GISGeography, "GIS Spatial Data Types: Vector vs Raster." [Online]. Available: http://gisgeography.com/spatial-data-types-vector-raster/. [Accessed: 25-Jul-2016]

[17] Veltman, N. and J. Ye, "A serverless boundary service from WNYC," 2014. [Online]. Available: https://source.opennews.org/en-US/articles/introducing-wherewolf/. [Accessed: 22-Jul-2016]. 


\section{Authors}

Puspa Miladin N S A Basid is a student of master program in Faculty of Computer Science, Universitas Brawijaya. He is also a member of Reserach Group of Media, Games \& Mobile. (email: puspamiladin2808@gmail.com)

Herman Tolle is a Head of Laboratory of Mobile Application Development Faculty of Computer Science, Universitas Brawijaya and Coordinator of Reserach Group of Media, Game \& Mobile. Also became a representative of Faculty of Computer Science in Team Quality Assurance Center (PJM) UB. (email: emang@ub.ac.id)

Fatwa Ramdani is a Coordinator of Research Group Geographic Information System Faculty of Computer Science, Universitas Brawijaya. (email: fatwaramdani@ub.ac.id)

Article submitted 21 December 2016. Published as resubmitted by the authors 13 February 2017. 